\title{
Maximal Surfaces in the 3-Dimensional Minkowski Space $L^{3}$
}

\author{
Osamu KOBAYASHI
}

\author{
Keio University
}

A surface in the 3-dimensional Minkowski space $L^{3}=\left(\boldsymbol{R}^{3}, d x^{2}+d y^{2}-d z^{2}\right)$ is called a space-like surface if the induced metric on the surface is a positive definite Riemannian metric. A space-like surface with vanishing mean curvature is called a maximal surface.

In this paper, we give the Weierstrass-Enneper representation formulas for maximal surfaces $(\S 1)$, and exhibit various examples $(\S 2)$. In particular, we determine the maximal surfaces which are rotation surfaces or ruled surfaces $(\S \S 3,4)$. In contrast with the case of minimal surfaces in the Euclidean 3-space, where a rotation minimal surface (resp. a ruled minimal surface) is locally congruent to a catenoid (resp. a helicoid), there are various types of maximal rotation or maximal ruled surfaces. For example, maximal Enneper's surfaces appear as rotation or ruled surfaces. In the last section, we give some explicit examples which show that the so-called Bernstein property does not hold, in general, without an ellipticity condition $(\S 5)$.

\section{§1. Weierstrass-Enneper formulas for maximal surfaces in $L^{8}$.}

For a space-like surface in $L^{3}$, the Gauss map is defined to be a mapping which assigns to each point of the surface the unit normal vector at the point. Therefore, its image can be regarded as contained in a space-like surface $H^{2}=\left\{(x, y, z) \in L^{3} ; x^{2}+y^{2}-z^{2}=-1\right\}$, which has constant negative curvature -1 with respect to the induced metric. We define a stereographic mapping $\sigma$ for $H^{2}$ in the following way

$$
\begin{aligned}
& \sigma: C \backslash\{|\zeta|=1\} \longrightarrow H^{2} ; \quad \zeta \longmapsto\left(\frac{-2 \operatorname{Re} \zeta}{|\zeta|^{2}-1}, \frac{-2 \operatorname{Im} \zeta}{|\zeta|^{2}-1}, \frac{|\zeta|^{2}+1}{|\zeta|^{2}-1}\right) \\
& \quad \text { and } \sigma(\infty)=(0,0,1) .
\end{aligned}
$$

That is, $\sigma(\zeta)$ is the intersection of $H^{2}$ and the line joining $(\operatorname{Re} \zeta, \operatorname{Im} \zeta, 0)$ Received June 21, 1982 
and the "north pole" $(0,0,1)$ of $H^{2}$. It is easy to see that $\sigma$ is conformal in the natural manner. Moreover, we can show that the Gauss map of a maximal surface is also conformal, which can be interpreted in the following form.

THEOREM 1.1 (Weierstrass-Enneper formula of 1st kind). Any maximal space-like surface in $L^{8}$ is represented as

$$
\psi(\zeta)=\operatorname{Re} \int\left(\frac{1}{2} f\left(1+g^{2}\right), \frac{\sqrt{-1}}{2} f\left(1-g^{2}\right),-f g\right) d \zeta, \quad \zeta \in D,
$$

where $D$ is a domain in $C$, and $f$ (resp. $g$ ) is a holomorphic (resp. meromorphic) function on $D$ such that $f g^{2}$ is holomorphic on $D$ and that $|g(\zeta)| \neq 1$ for $\zeta \in D$. Moreover,

(i) the Gauss map $G$ is given by $G(\zeta)=\sigma(g(\zeta))$, where $\sigma$ is a map defined by (1.1);

(ii) the induced metric is given by $d s=\left(\left.|f||1-| g\right|^{2} \mid / 2\right)|d \zeta|$;

(iii) the Gauss curvature of the surface is given by $K=\left\{4\left|\partial_{\xi} g\right| /|f|(1-\right.$ $\left.\left.|g|^{2}\right)^{2}\right\}^{2}$.

REMARK. Contrary to the case of minimal surfaces, a maximal surface in $L^{8}$ has non-negative curvature. In fact, by the Gauss equation for a maximal surface in $L^{3}$, we have $K=|h|^{2} / 2$, where $h$ is the second fundamental form.

Proof. Suppose that $\psi: D \rightarrow L^{3}$ is a maximal space-like surface. From the maximality, it is easy to see that $\Delta \psi=0$, where $\Delta$ is the Laplacian defined by the induced metric on $D$, which is a positive definite Riemannian metric. In particular, $D$ cannot be a closed surface. Hence, taking the universal covering of $D$, if necessary, we may assume that $D$ is a domain in $C$ and that $\psi$ is a conformal mapping. Set

$$
2 \partial_{5} \psi=\left(\varphi_{1}, \varphi_{2}, \varphi_{3}\right), \quad \text { where } \partial_{\zeta}=\frac{1}{2}\left(\partial_{u}-\sqrt{-1} \partial_{v}\right), \quad \zeta=u+\sqrt{-1} v .
$$

Then, the conformality of $\psi$ implies that $\varphi_{1}^{2}+\varphi_{2}^{2}-\varphi_{3}^{2}=0$, and $\Delta \psi=0$ implies that $\bar{\partial}_{\xi} \partial_{\xi} \psi=0$, i.e., $\varphi_{i}$ are holomorphic. Therefore, putting $f=\varphi_{1}-\sqrt{-1} \varphi_{2}$ and $g=-\varphi_{3} / f$, we have the formula (1.2). (Remark that $\varphi_{1}-\sqrt{-1} \varphi_{2} \equiv 0$ corresponds to the $(x, y)$-plane in $L^{3}$, but it can be obtained by putting $g \equiv 0$ and $f \equiv 1$ in (1.2).)

In $L^{3}$, we define the exterior product of vectors by $X \times Y=$ $-\left(\iota_{Y} \ell_{X} d x \wedge d y \wedge d z\right)^{*}$, where $\iota_{X}$ denotes the interior product with respect to $X$ and \# stands for the operation of raising indices by the metric 
$d x^{2}+d y^{2}-d z^{2}$. Here we choose the sign "-" so that $\partial_{x} \times \partial_{y}=\partial_{z}$ holds. Then a direct calculation yields that

$$
\partial_{u} \psi \times \partial_{v} \psi=\left\{|f|\left(1-|g|^{2}\right) / 2\right\}^{2} \sigma(g),
$$

which proves (i) and also (ii) because of the conformality of $\psi . K$ is easily calculated from (ii).

As an immediate consequence, we have

CoROLLARY 1.2. A piece of surface with constant positive curvature cannot be isometrically immersed in $L^{3}$ as a maximal surface.

Proof. It follows from (ii) and (iii) in the Theorem 1.1 that $\sqrt{K} d s^{2}$ is a flat metric if $K>0$ and hence that $d s^{2}$ cannot be a metric with positive constant curvature.

For later use, we need a variant of the above formula.

Corollary 1.3 (Weierstrass-Enneper formula of 2nd kind). Any maximal space-like surface in $L^{3}$ is represented as

$$
\psi(\zeta)=\operatorname{Re} \int\left(\frac{1}{2} f\left(g^{2}+1\right), \sqrt{-1} f g, \frac{1}{2} f\left(g^{2}-1\right)\right) d \zeta, \quad(\operatorname{Re} g \neq 0),
$$

and, the Gauss map $G$ is given by $G(\zeta)=\sigma((1-g) /(1+g))$.

Proof. Replace $f$ and $g$ in (1.2) by $f(1+g)^{2} / 2$ and $(1-g) /(1+g)$, respectively.

\section{§ 2. Examples.}

EXAMPLE 2.1. The simplest example of maximal surfaces in $L^{3}$ is a space-like plane, which is obtained by setting $g=$ constant in (1.2) or (1.3). It has been known that only plane is a complete maximal surface in $L^{3}$ ([1], [3]). A simple proof of this fact can be given by using the Weierstrass-Enneper formula (1.2) as follows: Let $\psi: D \rightarrow L^{3}$ be a complete maximal surface expressed in the form (1.2). We may then assume that $D$ is conformal to $C$ or the unit disk $\{|\zeta|<1\}$. In view of (i) in Theorem 1.1, we may assume also that $|g|<1$ on $D$. Suppose that $D$ is conformal to the unit disk. Then it is known [6; p. 67] that there is a divergent path $p(t)$ in $D$ such that $\int_{p(t)}|f(\zeta)||d \zeta|<\infty$, which shows, from (ii) of Theorem 1.1, that $\psi$ cannot be complete. Hence, $D$ is conformal 
to $C$. Then, by Liouville's theorem $g$ must be a constant function. Hence, $\psi$ is a plane.

EXAMPLE 2.2 (Enneper's surface of 1st kind). Set $f=2, g=\zeta$ and $D=C \backslash\{|\zeta|=1\}$ in (1.2). Then we have a maximal surface given by

$$
\psi(\zeta)=\left(u-u v^{2}+\frac{1}{3} u^{3},-v+u^{2} v-\frac{1}{3} v^{3}, v^{2}-u^{2}\right),
$$

where $\zeta=u+\sqrt{-1} v$. At first sight, we might find that it is very similar to the classical Enneper's minimal surface. As shown in the below, however, it is quite complicated.

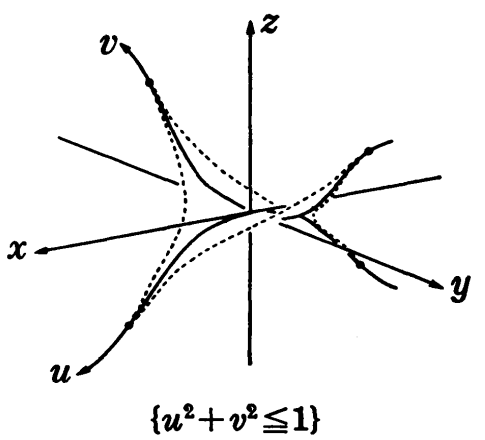

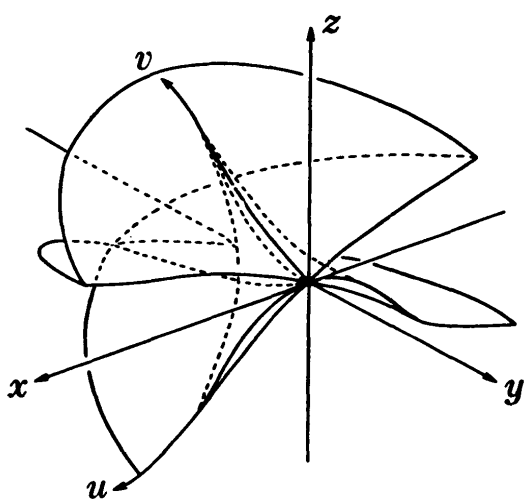

$\{y \leqq 0\}$

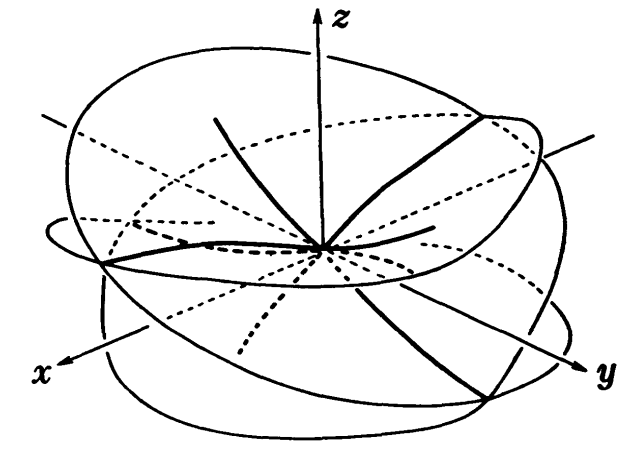

$\left\{v^{2}-\frac{1}{3} u^{2} \geqq 1\right\} \cup\left\{u^{2}-\frac{1}{3} v^{2} \geqq 1\right\}$

FIGURE 1. Enneper's surface of 1st kind.

EXAMPLE 2.3 (Enneper's surface of 2nd kind). This is given by putting $f=2 a, g=\zeta$ in (1.3), where $a$ is a non-zero real constant. An explicit formula is given as follows:

$\psi(\zeta)=a\left(u-u v^{2}+\frac{1}{3} u^{3},-2 u v,-u-u v^{2}+\frac{1}{3} u^{3}\right), \quad \zeta=u+\sqrt{-1} v, \quad u \neq 0$.

This surface is a rotation surface with light-like axis $(1,0,1)$, which can 
be seen from the following expression (cf. §3):

$$
\psi(\zeta)=\left(\begin{array}{ccc}
1-\frac{1}{2} v^{2} & v & \frac{1}{2} v^{2} \\
-v & 1 & v \\
-\frac{1}{2} v^{2} & v & 1+\frac{1}{2} v^{2}
\end{array}\right)\left(\begin{array}{c}
a u+\frac{a}{3} u^{3} \\
0 \\
-a u+\frac{a}{3} u^{3}
\end{array}\right), \quad u \neq 0
$$

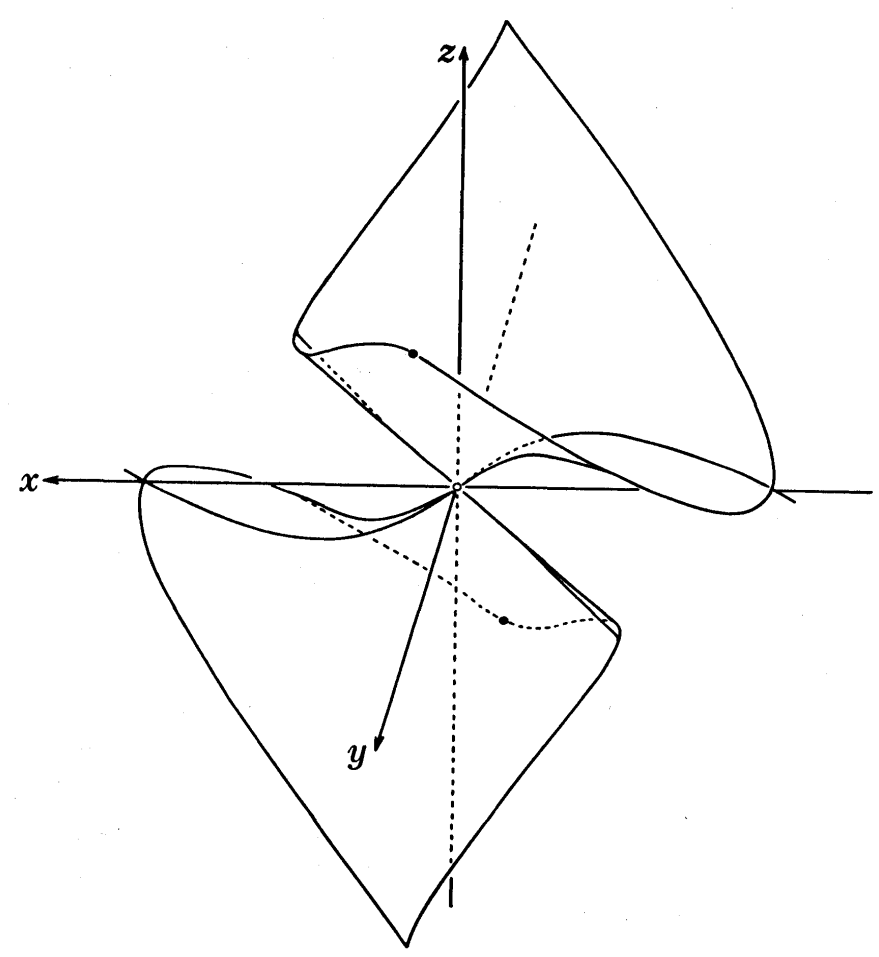

FigurE 2. Enneper's surface of 2nd kind.

EXAMPLE 2.4 (Conjugate of Enneper's surface of 2nd kind). Analogous to the definition of the conjugate surface of a minimal surface in the Euclidean space, we define the conjugate surface of Enneper's surface of 2 nd kind by putting $f=2 a \sqrt{-1}$ and $g=\zeta$ in (1.3). Then we have

$$
\begin{aligned}
\psi(\zeta) & =a\left(-v-u^{2} v+\frac{1}{3} v^{3}, v^{2}-u^{2}, v-u^{2} v+\frac{1}{3} v^{3}\right) \\
& =a\left(-v+\frac{1}{3} v^{3}, v^{2}, v+\frac{1}{3} v^{s}\right)-a u^{2}(v, 1, v), \quad u \neq 0 .
\end{aligned}
$$

In consequence, this is a ruled surface. 


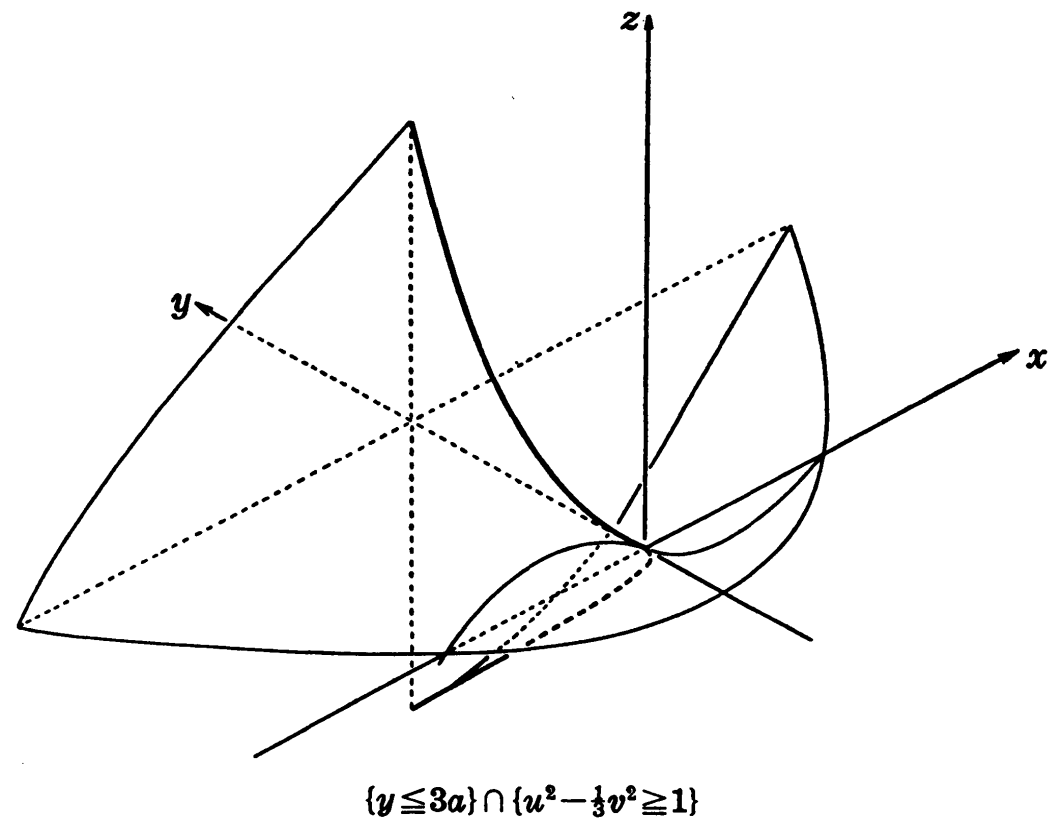

Figure 3. Conjugate of Enneper's surface of 2nd kind.

EXAMPLE 2.5 (Catenoid of 1st kind). This is a rotation surface defined by $x^{2}+y^{2}-a^{2} \sinh ^{2}(z / a)=0,(z \neq 0)$, where $a$ is a non-zero real. In view of the Weierstrass-Enneper formula, it is given by putting $f=a \zeta^{-2}$, $g=\zeta$ in (1.2).

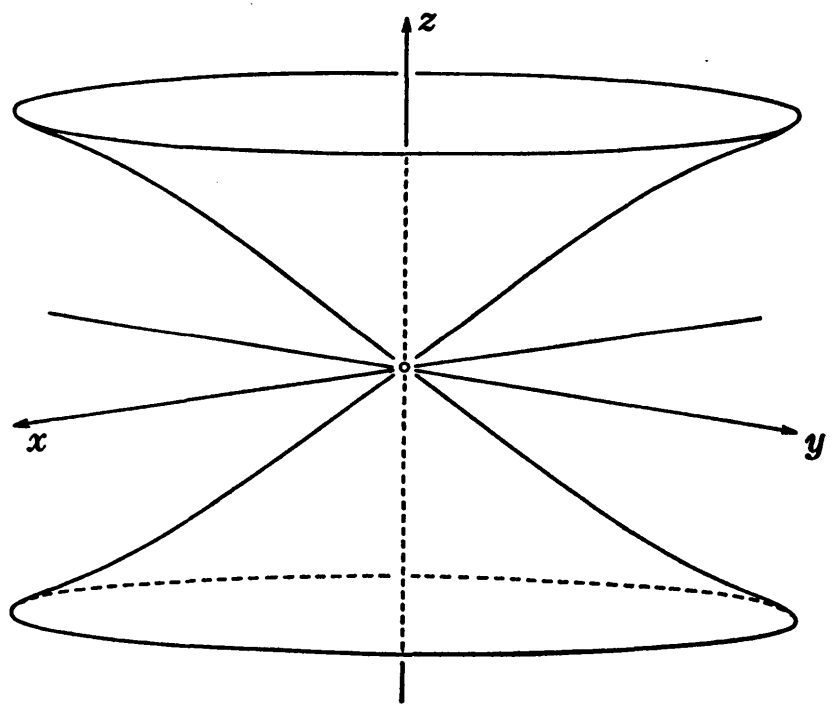

FIGURE 4. Catenoid of 1st kind.

EXAMPLE 2.6 (Helicoid). The conjugate surface of a catenoid of 1st kind, that is, the surface defined by setting $f=\sqrt{-1} a \zeta^{-2}, g=\zeta$ in (1.2), 
is given by

$$
\psi(\zeta)=(0,0, a \theta)+a \cosh \log r(-\sin \theta, \cos \theta, 0), \quad \zeta=r e^{\sqrt{-1} \theta}, \quad(r \neq 1) .
$$

Note that this is an open subset of the usual helicoid; $x \cos (z / a)+$ $y \sin (z / a)=0$. Hence, it is also a minimal surface with respect to the metric $d x^{2}+d y^{2}+d z^{2}$. Conversely, this property characterizes the helicoid (§4).

EXAMPLe 2.7 (Catenoid of 2nd kind). This is a rotation surface defined by putting $f=a \zeta^{-2}, g=\zeta$ in (1.3):

$$
\psi(\zeta)=\left(\begin{array}{ccc}
\cosh \log r & 0 & \sinh \log r \\
0 & 1 & 0 \\
\sinh \log r & 0 & \cosh \log r
\end{array}\right)\left(\begin{array}{c}
0 \\
-a \theta \\
a \cos \theta
\end{array}\right), \quad \zeta=r e^{\gamma-1 \theta}, \quad(\cos \theta \neq 0)
$$

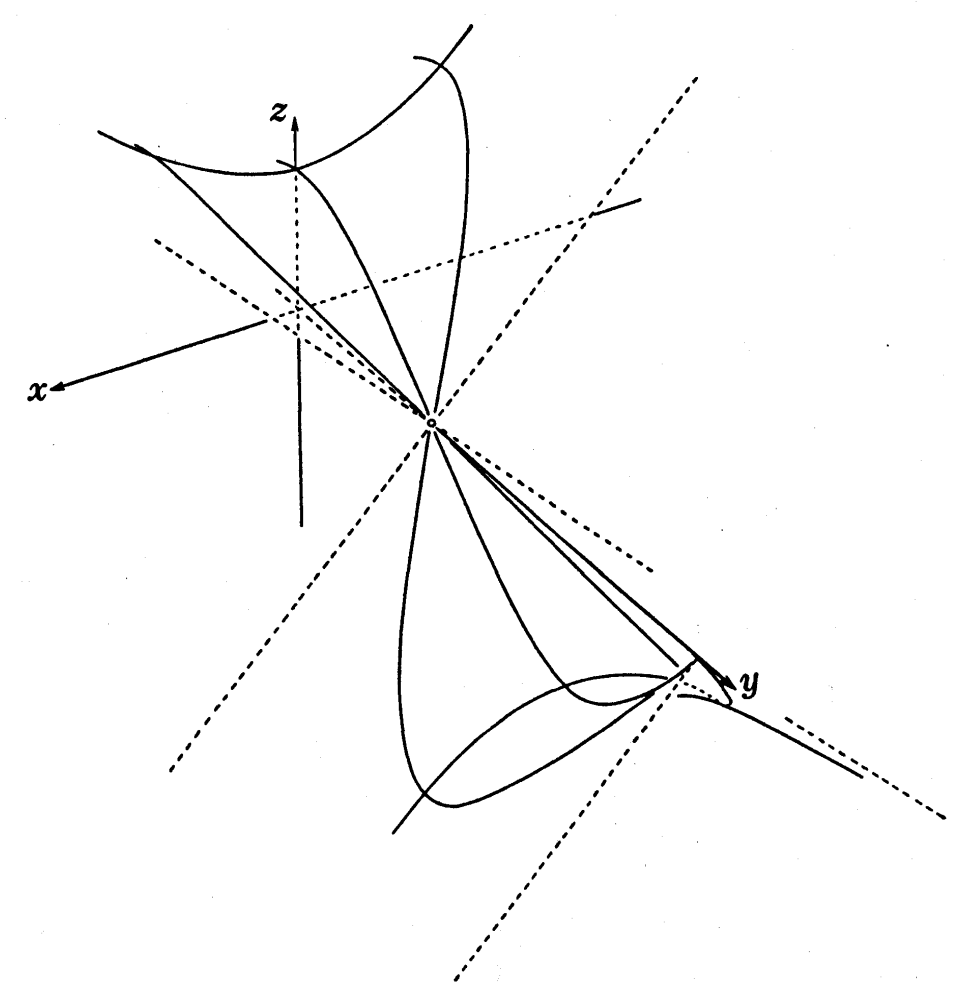

Figure 5. Catenoid of 2nd kind.

EXAMPLE 2.8 (Helicoid of 2nd kind). This is a ruled surface defined by $z+x \tanh (y / a)=0\left(x^{2} \leqq a^{2} \cosh ^{2}(y / a)\right)$, which corresponds to $f=\sqrt{-1} a \zeta^{-2}$ and $g=\zeta$ in (1.3).

EXAMPLe 2.9 (Scherk's surface of 1st kind). This is a maximal surface 
defined by $z=\log \cosh y-\log \cosh x, \quad\left(\cosh ^{-2} x+\cosh ^{-2} y>1\right)$, which is obtained by putting $f=4\left(1-\zeta^{4}\right)^{-1}$ and $g=\zeta$ in (1.2).

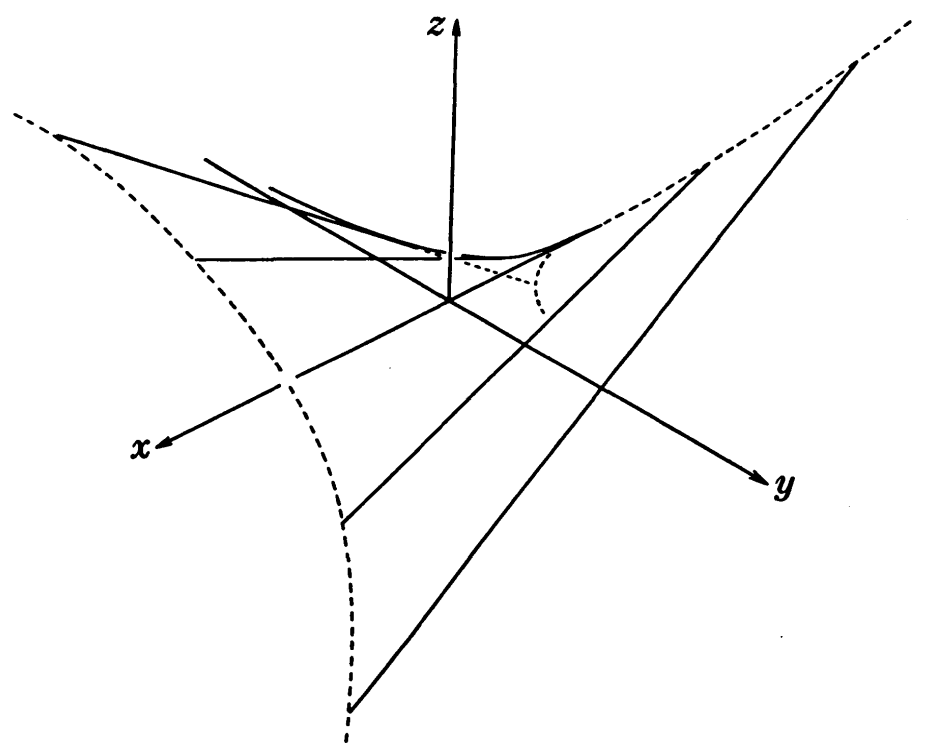

Figure 6. Helicoid of 2nd kind.

\section{§3. Rotation surfaces.}

The purpose of this section is to determine the maximal rotation surfaces in $L^{3}$. A surface is called a rotation surface with axis $l$ if it is invariant under the action of the group of motions in $L^{8}$ which fix each point of the line $l$.

THEOREM 3.1. Every maximal rotation surface in $L^{3}$ is congruent to a part of one of the following:

(i ) $(x, y)-p l a n e ;$

(ii) catenoid of 1 st kind;

(iii) catenoid of 2 nd kind;

(iv) Enneper's surface of 2 nd kind.

Proof. The $(x, y)$-plane is obviously a rotation surface with timelike axis, and every space-like plane is congruent to it. So, in the following, we assume that the given maximal rotation surface is not a plane.

If the axis is time-like (resp. space-like), we may suppose that the axis is the $z$-axis (resp. $y$-axis), since every time-like (resp. space-like) unit vector is transformed to $(0,0,1)$ (resp. $(0,1,0)$ ) by a Lorentz transformation. Then the surface is expressed as follows: 
(3.1) $\quad \psi(s, t)=(f(t) \cos s, f(t) \sin s, t)$ if the axis is time-like;

(3.2) $\psi(s, t)=(g(t) \sinh s, t, g(t) \cosh s)$ if the axis is space-like.

The maximal surface equations are then given by

$$
\begin{array}{lll}
f \ddot{f}=\dot{f}^{2}-1, & \dot{f}^{2}-1>0 & \text { for }(3.1) ; \\
g \ddot{g}=\dot{g}^{2}-1, & \dot{g}^{2}-1<0 & \text { for }(3.2) .
\end{array}
$$

Hence, we have $f(t)=a^{-1} \sinh (a t+b)$ and $g(t)=a^{-1} \cos (a t+b)$, where $a$ and $b$ are integral constants. Thus, the surface is locally congruent to a catenoid of 1st kind or a catenoid of 2 nd kind according to that the axis is time-like or space-like.

If the axis is light-like, we may assume that it is $R \cdot(1,0,1)$. Note that the subgroup of the Lorentz group which fixes $(1,0,1)$ is

$$
\left\{\left(\begin{array}{ccc}
1-\frac{s^{2}}{2} & s & \frac{s^{2}}{2} \\
-s & 1 & s \\
-\frac{s^{2}}{2} & s & 1+\frac{s^{2}}{2}
\end{array}\right) ; s \in R\right\}
$$

Hence, the surface can be written as

$$
\psi(s, t)=\left(\begin{array}{ccc}
1-\frac{s^{2}}{2} & s & \frac{s^{2}}{2} \\
-s & 1 & s \\
-\frac{s^{2}}{2} & s & 1+\frac{s^{2}}{2}
\end{array}\right)\left(\begin{array}{c}
h(t)+t \\
0 \\
h(t)-t
\end{array}\right)
$$

The maximal surface equation for (3.5) is given by

$$
t \ddot{h}-2 \dot{h}=0, \quad t \neq 0, \dot{h}>0 .
$$

Thus, we have the solution $h(t)=a t^{3}+b, a>0$, which shows that the surface is Enneper's surface of 2nd kind.

\section{§4. Ruled surfaces.}

As for maximal ruled surfaces, we have the following:

THEOREM 4.1. Every maximal ruled surface in $L^{3}$ is congruent to a part of one of the following:

(i ) (x,y)-plane; 
(ii) helicoid;

(iii) helicoid of 2 nd kind;

(iv) conjugate of Enneper's surface of 2 nd kind.

Proof. Every space-like ruled surface can be written as

$$
\begin{aligned}
& \psi(s, t)=c(t)+s n(t), \\
& n(t) \cdot n(t)=\dot{c}(t) \cdot \dot{c}(t)=1, \quad \dot{c}(t) \cdot n(t)=0,
\end{aligned}
$$

where $c(t)$ is a space-like curve in $L^{8}$ with arclength parameter and $n(t)$ is a unit normal vector field along $c(t)$. Note that $n(t)$ is an asymptotic vector field on the surface and that $\dot{c}(t)+s \dot{n}(t)$ is perpendicular to $n(t)$. It follows from the maximality that $\dot{c}(t)+\boldsymbol{s} \dot{n}(t)$ is an asymptotic direction. In particular, putting $s=0$, we can see that $n(t)$ is the principal normal vector of $c(t)$. Hence, we need only to determine the curve $c(t)$ to get the surface (4.1). Denoting by $b(t)$ the binormal of $c(t)$, we have the Frenet-Serret formula:

$$
\begin{array}{ll}
\ddot{c}(t)=\kappa(t) n(t), & \dot{n}(t)=-\kappa(t) \dot{c}(t)+\tau(t) b(t), \\
\dot{b}(t)=\tau(t) n(t), &
\end{array}
$$

where $\kappa$ and $\tau$ are curvature and torsion of $c(t)$, respectively. Hence,

$$
\dot{c}(t)+s \dot{n}(t)=(1-s \kappa(t)) \dot{c}(t)+s \tau(t) b(t) ;
$$

$$
\ddot{c}(t)+s \ddot{n}(t)=\left(\kappa(t)-s \kappa(t)^{2}+s \tau(t)^{2}\right) n(t)+s(-\dot{\kappa}(t) \dot{c}(t)+\dot{\tau}(t) b(t)) .
$$

Since $\dot{c}+s \dot{n}$ is an asymptotic direction, $\ddot{c}+s \ddot{n}$ is tangent to the surface. That is, $s(-\dot{\kappa}(t) \dot{c}(t)+\dot{\tau}(t) b(t))$ must be parallel to $(1-8 \kappa(t)) \dot{c}(t)+\tau(t) b(t)$ for any $s$ and $t$. Hence, $\kappa$ and $\tau$ are constant.

Then, if $|\kappa|>|\tau|>0$ (resp. $|\tau|>|\kappa|>0), \tilde{c}(t)=c(t)+\left(\kappa /\left(\kappa^{2}-\tau^{2}\right)\right) n(t)$ is a time-like (resp. space-like) line by (4.3) and (4.4). Therefore, from (4.2), we can see that $c(t)$ is congruent to

$$
\begin{array}{r}
\left(\frac{\kappa}{\kappa^{2}-\tau^{2}} \cos \sqrt{\kappa^{2}-\tau^{2}} t, \frac{\kappa}{\kappa^{2}-\tau^{2}} \sin \sqrt{\kappa^{2}-\tau^{2}} t, \frac{\tau}{\sqrt{\kappa^{2}-\tau^{2}}} t\right) \\
\text { if }|\kappa|>|\tau|>0 ;
\end{array}
$$

or

$$
\begin{array}{r}
\left(\frac{\kappa}{\tau^{2}-\kappa^{2}} \cosh \sqrt{\tau^{2}-\kappa^{2}} t, \frac{\tau}{\sqrt{\tau^{2}-\kappa^{2}}} t, \frac{\kappa}{\tau^{2}-\kappa^{2}} \sinh \sqrt{\tau^{2}-\kappa^{2}} t\right) \\
\text { if }|\tau|>|\kappa|>0 .
\end{array}
$$

In each case, the surface defined by (4.1) is congruent to a part of 
helicoid or that of 2nd kind.

If $|\tau|=|\kappa| \neq 0$, we have $\ddot{n}=0$ by (4.2), hence $\dddot{c}=0$. That is, $c(t)$ is a polynomial of degree 3 . Then, we have the conjugate of Enneper's surface of 2 nd kind.

Now, we give a characterization of the helicoid:

THEOREM 4.2. Except for the plane, only the helicoid is a maximal surface in $L^{8}=\left(\boldsymbol{R}^{3}, d x^{2}+d y^{2}-d z^{2}\right)$ which is minimal surface with respect to the Riemannian metric $d x^{2}+d y^{2}+d z^{2}$.

Proof. Locally, a space-like surface is always a graph of $z=f(x, y)$. The maximal surface equation for $z=f(x, y)$ is

$$
\left(1-f_{x}^{2}\right) f_{y y}+2 f_{x} f_{y} f_{x y}+\left(1-f_{y}^{2}\right) f_{x x}=0 \text {. }
$$

On the other hand, the minimal surface equation for $z=f(x, y)$ is

$$
\left(1+f_{x}^{2}\right) f_{y y}-2 f_{x} f_{y} f_{x y}+\left(1+f_{y}^{2}\right) f_{x x}=0 .
$$

Suppose that $f$ satisfies (4.7) as well as (4.8). Then we get

$$
-f_{x}^{2} f_{y y}+2 f_{x} f_{y} f_{x y}-f_{y}^{2} f_{x x}=0 \text {. }
$$

Put $X=\left(-f_{y}, f_{x}\right)$, which is a vector field tangent to $\{f=$ constant $\}$ in $(x, y)$-plane. Then, we have

$$
\operatorname{det}\left(X, \nabla_{X} X\right)=-f_{x}^{2} f_{y y}+2 f_{x} f_{y} f_{x y}-f_{y}^{2} f_{x x} .
$$

This, together with (4.9), implies that the integral curves of $X$ in $R^{2}$ are lines. Since $f$ is constant on each of these integral curves, it follows that the surface $z=f(x, y)$ is a ruled surface. It is well-known that a ruled minimal surface in $R^{3}$ is a plane or a helicoid. Thus, the result follows.

§5. Entire solutions to the maximal hypersurface equations.

It is known that there are no non-flat complete non-parametric maximal hypersurfaces in $L^{n+1}=\left(\boldsymbol{R}^{n+1},\left(d x^{1}\right)^{2}+\cdots+\left(d x^{n}\right)^{2}-\left(d x^{n+1}\right)^{2}\right)$ ([3]). In other words, except for linear functions, there are no entire solutions to

$$
\left(1-\sum_{i=1}^{n}\left(\frac{\partial f}{\partial x^{i}}\right)^{2}\right) \sum_{j=1}^{n} \frac{\partial^{2} f}{\left(\partial x^{j}\right)^{2}}+\sum_{i, j=1}^{n} \frac{\partial f}{\partial x^{i}} \frac{\partial f}{\partial x^{j}} \frac{\partial^{2} f}{\partial x^{i} \partial x^{j}}=0
$$

and 


$$
1-\sum_{i=1}^{n}\left(\frac{\partial f}{\partial x^{i}}\right)^{2}>0 \text {. }
$$

Here, (5.2) is a condition which assures of the hypersurface $x^{n+1}=$ $f\left(x^{1}, \cdots, x^{n}\right)$ being space-like, and also which makes the equation (5.1) elliptic. However, if we do not assume the condition (5.2), there are non-trivial entire solutions to (5.1). For instance:

Proposition 5.1. The following are entire solutions to (5.1) for $n \geqq 2$ :

(i) $f=x^{1} \tanh x^{2}$; (ii) $f=\log \cosh x^{1}-\log \cosh x^{2}$.

Proof. Direct calculations.

Similar phenomenon occurs in geometry of affine minimal hypersurfaces. Consider $R^{n+1}$ as an affine space with the canonical volume element $d x^{1} \wedge d x^{2} \wedge \cdots \wedge d x^{n+1}$ and the canonical flat affine connection. A hypersurface in $R^{n+1}$ is said to be non-degenerate if, at each point of the hypersurface, the osculating quadratic hypersurface is proper. For a non-degenerate hypersurface, there is a concept of mean curvature which is defined by the volume element and the connection of $\boldsymbol{R}^{n+1}$, without using any metric of $\boldsymbol{R}^{n+1}$. If the hypersurface is a graph $x^{n+1}=f\left(x^{1}, \cdots, x^{n}\right)$, then the non-degeneracy condition and the affine minimal hypersurface equation are given respectively by

$$
\operatorname{det}\left(f_{i j}\right) \neq 0
$$

and

$$
(n+2) \sum_{i, k}\left((\log |H|)_{i} f^{i k}\right)_{k}+\sum_{i, k} f^{i k}(\log |H|)_{i}(\log |H|)_{k}=0,
$$

where $f_{i j}=\left(\partial^{2} f / \partial x^{i} \partial x^{j}\right), H=\operatorname{det}\left(f_{i j}\right)$ and $\left(f^{i j}\right)$ is the inverse matrix of $\left(f_{i j}\right)$. There has been posed a problem [4] whether any entire solution to (5.4) is affinely equivalent to

$$
f=\sum_{i=1}^{n}\left(x^{i}\right)^{2},
$$

provided $\left(f_{i j}\right)$ is positive definite. For $n=2$, this has been answered affirmatively [2]. Now, we shall show that there are entire solutions to (5.3) and (5.4) which are not of the form (5.5), if we do not assume that $\left(f_{i j}\right)$ is positive definite.

Proposition 5.2. $f=x^{1} \tanh x^{2}+\sum_{i=8}^{n}\left(x^{i}\right)^{2}$ is an entire solution to (5.3) and (5.4) for $n \geqq 2$. 
Proof. A direct calculation. 2.9 .

Propositions 5.1 and 5.2 should be compared with Examples 2.8 and

\section{References}

[1] E. Calabi, Examples of Bernstein problems for some non-linear equations, Proc. Symp. Pure Math., 15 (1970), 223-230.

[2] E. CALABI, Hypersurfaces with maximal affinely invariant area, Amer. J. Math., 104 (1981), 91-126.

[ 3 ] S.-Y. ChEng and S.-T. YAU, Maximal space-like hypersurfaces in the Lorentz-Minkowski spaces, Ann. of Math., 104 (1976), 407-419.

[4] S.-S. CherN, Affine minimal hypersurfaces, Proc. of US-Japan Seminar on Minimal Submanifolds, Tokyo 1978, 17-30.

[5] B. Lawson, Lectures on Minimal Submanifolds, vol. 1, Publish or Perish, Inc., 1980.

[6] R. Osserman, A Survey of Minimal Surfaces, Van Nostrand, New York, 1969.

Present Address:

DePartment OF Mathematics

KEIO UNIVERSITY

HIYOSHI, KOHOKU-KU

YoKoHAMA 223 